\title{
CONSUMO DE ALCOHOL Y GÉNERO EN LA POBLACIÓN ADOLESCENTE ESCOLARIZADA DEL PERÚ: EVOLUCIÓN Y RETOS DE INTERVENCIÓN
}

\author{
William Cabanillas-Rojas (iㅣ, \\ ${ }^{1}$ Observatorio Peruano de Drogas. Comisión Nacional para el Desarrollo y Vida sin Drogas (DEVIDA), Lima, Perú. \\ a Licenciado en Psicología.
}

\section{RESUMEN}

El involucramiento en el uso de alcohol representa un riesgo significativo para el desarrollo mental y físico de los adolescentes. El objetivo de este artículo es describir la evolución del consumo de alcohol en escolares hombres y mujeres de nivel secundario del Perú durante el periodo 2007-2017, haciendo énfasis en los hallazgos sobre la reducción de las diferencias en las magnitudes de uso entre ambos géneros y la información reciente sobre mayor prevalencia en la población femenina adolescente. Se proponen recomendaciones dirigidas a la ampliación de cobertura y calidad de los programas preventivos en el ámbito escolar, adaptación de contenidos y estrategias preventivas sensibles al género, conformación de mecanismos articulados de detección temprana, derivación e intervención breve, elaboración de guías y protocolos específicos de tratamiento para adolescentes, y fortalecimiento de la articulación intersectorial para la reducción del consumo del alcohol en menores de edad.

Palabras clave: Alcohol; Género; Adolescente; Perú; Escuela (fuente: DeCS BIREME).

\section{ALCOHOL CONSUMPTION AND GENDER IN THE ADOLESCENT SCHOOL POPULATION OF PERU: EVOLUTION AND INTERVENTION CHALLENGES}

\begin{abstract}
The involvement in the use of alcohol represents a significant risk for the mental and physical development of adolescents. The objective of this article is to describe the evolution of alcohol consumption in male and female secondary school students in Peru during the 2007-2017 period, emphasizing the findings on the reduction of differences in the usage magnitudes between both genders and recent information on high prevalence in the adolescent female population. Recommendations are proposed aimed at expanding coverage and quality of preventive programs in the school, adaptation of gender-sensitive content and preventive strategies, creation of articulated mechanisms for early detection, referral and brief intervention, development of specific guidelines and protocols for treatment for adolescents, and strengthening of the intersectoral articulation for the reduction of alcohol consumption in adolescents.
\end{abstract}

Keywords: Alcohol; Gender; Adolescent; Peru; School (source: MeSH NLM).

Citar como: Cabanillas-Rojas W. Consumo de alcohol y género en la población adolescente escolarizada del Perú: evolución y retos de intervención. Rev Peru Med Exp Salud Publica. 2020;37(1):148-54. doi: https://doi.org/10.17843/rpmesp.2020.371.5151

Correspondencia: William Cabanillas-Rojas; wcabanillas@devida.gob.pe

Recibido: $23 / 01 / 2020$ Aprobado: 26/02/2020 En línea: 23/03/2020

\section{INTRODUCCIÓN}

El consumo de alcohol es un importante factor de riesgo para diversas consecuencias adversas durante todo el ciclo vital; sin embargo, en la adolescencia cobra relevancia especial por sus efectos negativos en el desarrollo físico y mental. El involucramiento en el uso de bebidas alcohólicas por parte de los adolescentes se asocia, entre otros, con efectos negativos en el desarrollo cerebral $^{(1)}$, menor rendimiento académico ${ }^{(2)}$, relaciones sexuales de riesgo ${ }^{(3)}$, conductas delictivas ${ }^{(4)}$, lesiones y accidentes ${ }^{(5)}$, suicidio ${ }^{(6)}$, así como con trastornos por abuso de alcohol en la edad adulta ${ }^{(7)}$. Estos riesgos psicosociales y sanitarios vinculados al uso de alcohol justifican la necesidad de emprender esfuerzos de salud pública para los adolescentes, considerando datos epidemiológicos y la utilización de intervenciones basadas en la evidencia.

A nivel mundial, 155 millones de adolescentes consumen alcohol, siendo el continente americano el segundo en magnitud ${ }^{(8)}$. En el Perú, el alcohol es la droga más consumida entre 
los adolescentes. Desde el establecimiento de mediciones nacionales estandarizadas y comparables en la población escolar de nivel secundario, el consumo de alcohol ha registrado las mayores prevalencias. Recientemente, se ha estimado que el 29,5\% de los escolares de nivel secundario (alrededor de 438000 adolescentes) refiere haber usado alcohol alguna vez en la vida y el 17,5\% (aproximadamente 259000 estudiantes) reporta haber usado alcohol en los últimos doce meses ${ }^{(9)}$.

Esta primera aproximación epidemiológica da cuenta sobre las significativas magnitudes de consumo de bebidas alcohólicas en los escolares de nivel secundario. No obstante, las características del uso de alcohol según género en este grupo de adolescentes y sus alternativas de intervención han sido aspectos poco explorados en nuestro país. En ese sentido, es importante destacar la necesidad de efectuar un análisis en función del género que facilite los procesos de diseño e implementación de políticas y actuaciones, tanto preventivas como terapéuticas.

Partiendo de un marco explicativo sobre el consumo de alcohol, se identifican factores de riesgo que ejercen efectos diferenciados en mujeres y hombres adolescentes. Por ejemplo, los hombres adolescentes presentan menor reactividad al alcohol, requiriendo mayores dosis de esa sustancia que las mujeres adolescentes. Asimismo, los hombres adolescentes pueden estar más influenciados por el consumo parental de alcohol y pueden ser más susceptibles a la influencia de pares. Se ha evidenciado también que el monitoreo parental es un factor de protección más influyente en las mujeres adolescentes $^{(10)}$.

A este panorama debe agregarse que la población femenina presenta una vulnerabilidad específica $\left.{ }^{(11,12)}: 1\right)$ son más susceptibles que los hombres a problemas asociados con el consumo de alcohol; 2) progresan más rápidamente hacia el consumo problemático de alcohol en comparación a los hombres; 3 ) tienden a ser más susceptibles que los hombres a los problemas de salud asociados con el consumo de alcohol; 4) tienen una progresión más rápida y grave hacia consumos dependientes; y 5) son más vulnerables a recibir agresiones sexuales.

Con base en lo expuesto, el objetivo de este artículo es analizar las magnitudes y características del consumo de alcohol según género en la población escolar de nivel secundario del Perú. Del mismo modo, se exploran las implicancias de la reducción en las diferencias de consumo de alcohol en hombres y mujeres; se proponen además recomendaciones de política e intervención preventiva y terapéutica para la población adolescente. Para este fin, se han revisado publicaciones especializadas, documentos institucionales y la información de los reportes 2007, 2009, 2012 y 2017 del Estudio Nacional sobre Prevención y Consumo de Drogas en Estudiantes de Secundaria elaborados por la Comisión Nacional para el Desarrollo y Vida sin Drogas (DEVIDA).

\section{MAGNITUDES Y CARACTERÍSTICAS DEL CONSUMO DE ALCOHOL SEGÚN GÉNERO EN LA POBLACIÓN ADOLESCENTE}

\section{Evolución de la prevalencia anual de consumo de alcohol en la población escolar de nivel secunda- rio según género}

En la última década, el consumo de alcohol entre los escolares de nivel secundario ha presentado un comportamiento tendiente a la disminución (Figura 1). El análisis según género, comparando el 2007 y 2017, revela que la prevalencia anual de consumo de alcohol ha disminuido $50 \%$ en hombres y $32 \%$ en mujeres. Sin embargo, a pesar de este aparente panorama alentador, se identifica en el 2017 magnitudes de uso de alcohol que expresarían la ocurrencia de una convergencia de género. Por convergencia de género entenderemos a que las diferencias del consumo de alcohol en mujeres y hombres adolescentes han disminuido a lo largo del tiempo.

Al desagregar la prevalencia anual de consumo de alcohol según género, se observan leves diferencias hasta el 2012. Durante el periodo 2009-2012 el descenso del consumo de alcohol fue menor en el grupo femenino en comparación al grupo masculino. Para el 2017 se registra un cambio importante en el uso de alcohol entre los escolares de nivel secundario ${ }^{(9)}$, siendo superior la prevalencia anual de las mujeres frente a los hombres (19,5\% y 15,5\%, respectivamente).

La identificación de mayores prevalencias anuales de consumo de alcohol en las escolares del Perú guarda correspondencia con la evidencia que sugiere una reducción de las diferencias de género en el consumo de alcohol ${ }^{(13)}$, como también a la información epidemiológica de otros países de la región ${ }^{(14)}$. Al analizar las magnitudes recientes de consumo de alcohol según género en función de variables sociodemográficas (Tabla 1), se observa que el grupo femenino obtiene mayores prevalencias anuales de consumo de alcohol, excepto en las estudiantes de 17 a 20 años de edad y quinto

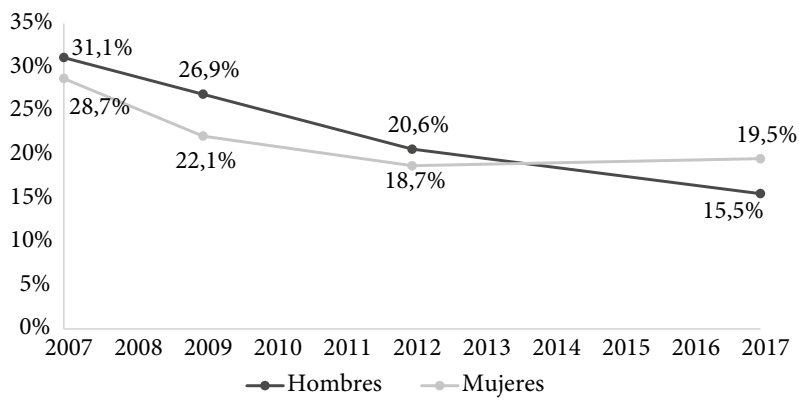

Fuente: Estudio Nacional sobre Prevención y Consumo de Drogas en Estudiantes de Secundaria, 2007-2017

Figura 1. Evolución de la prevalencia anual de consumo de alcohol en la población escolar de nivel secundario según género, 2007-2017 
Tabla 1. Prevalencia anual del consumo de alcohol en mujeres y hombres según variables sociodemográficas

\begin{tabular}{lcc}
\hline \multirow{2}{*}{ Características } & \multicolumn{2}{c}{ Prevalencia (IC 95\%) } \\
\cline { 2 - 3 } $\begin{array}{l}\text { Total } \\
\text { Grupo de edad (años) }\end{array}$ & Hombres \\
$\quad 11$ a 13 & & $15,5(14,3-16,7)$ \\
$\quad 14$ a 16 & $9,5(8,4-10,8)$ & $6,1(5,2-7,1)$ \\
$\quad$ 17 a 20 & $25,9(24,7-29,3)$ & $20,6(18,8-22,5)$ \\
Año de estudio & & $35,5(30,5-40,8)$ \\
$\quad$ Primero & $6,9(5,0-9,4)$ & $4,7(3,7-5,8)$ \\
$\quad$ Segundo & $13,1(11,1-15,3)$ & $8,1(6,6-9,8)$ \\
$\quad$ Tercero & $19,8(16,7-23,3)$ & $13,7(12,0-15,5)$ \\
$\quad$ Cuarto & $25,8(22,2-29,7)$ & $20,6(17,0-24,6)$ \\
$\quad$ Quinto & $34,4(30,7-38,3)$ & $35,3(31,2-39,6)$ \\
Tipo de colegio & & \\
$\quad$ Público & $16,0(14,6-17,6)$ & $13,7(12,6-14,9)$ \\
$\quad$ Privado & $25,4(22,8-28,1)$ & $18,2(16,0-20,6)$ \\
Ámbito geográfico & & \\
$\quad$ Lima Metropolitana & $21,7(18,9-24,7)$ & $16,0(13,9-18,3)$ \\
$\quad$ Resto del país & $17,9(16,6-19,2)$ & $15,0(13,9-16,3)$ \\
\hline
\end{tabular}

Fuente: DEVIDA - Estudio Nacional sobre Prevención y Consumo de Drogas en Estudiantes de Secundaria 2017

año de secundaria. Tanto mujeres como hombres presentan un patrón incremental de consumo alcohol a medida que se avanza en los grados de estudio. Se advierte también que ambos sexos comparten prevalencias anuales significativas de consumo de alcohol en el grupo de edad de 11 a 13 años y primer año de secundaria; por consiguiente, se identifica uso precoz de alcohol en mujeres y hombres.

Además del reporte de uso de alcohol, se identificó una importante proporción de estudiantes que presentan episodios de embriaguez e indicadores de consumo intensivo y consumo problemático. Estos patrones de utilización de bebidas alcohólicas preocupan por sus efectos negativos. La investigación sugiere que los episodios de embriaguez en la adolescencia incrementan el riesgo de mortalidad ${ }^{(15)}$ e involucramiento en comportamientos riesgosos como el consumo de drogas ilegales, accidentes, participación en acciones de violencia y bajo desempeño académico ${ }^{(16)}$.

Lo mismo ocurre con el beber excesivo o binge drinking (patrón de consumo de alcohol caracterizado por la ingesta de cinco o más bebidas alcohólicas en una única ocasión en las dos últimas semanas). La investigación con adolescentes muestra de forma consistente que el beber excesivo incrementa la posibilidad de ocurrencia de violencia interpersonal, lesiones intencionales y no intencionales, menor desempeño académico, comportamientos sexuales de riesgo, entre otros ${ }^{(17)}$. También existe evidencia de que el beber excesivo en la adolescencia se relaciona con el desarrollo de trastornos por consumo de alcohol en la adultez ${ }^{(18)}$.
La Tabla 2 muestra que mujeres y hombres presentan porcentajes similares de reporte embriaguez de uno a dos días. En contraste, el reporte de embriaguez de tres a más días es superior en el grupo masculino. De otro lado, se observa una leve diferencia entre hombres y mujeres en el consumo intensivo de alcohol reportado al menos una vez. No obstante, el grupo masculino reporta un mayor número de episodios. En cuanto al consumo problemático de alcohol, que hace referencia a un patrón de consumo que genera daños a la salud y afectación psicosocial, se evidencian proporciones similares en ambos géneros. Estos datos tienen especial relevancia para reconocer la existencia de un patrón desadaptativo de consumo de alcohol en un grupo significativo de escolares, involucrando de forma similar a mujeres y hombres.

En general, estos hallazgos nos permiten redefinir nuestra comprensión sobre el consumo de alcohol en la población adolescente, cuestionando la visión androcéntrica sobre esta problemática, así como a los modelos tradicionales de masculinidad y feminidad. Como se ha podido apreciar, las cercanías en las magnitudes de uso de bebidas alcohólicas en hombres y mujeres también comprenden patrones riesgosos de consumo.

Tabla 2. Reporte de embriaguez, beber excesivo de alcohol (usuarios de último mes) y consumo problemático de alcohol (usuarios de último año) en escolares de nivel secundario según género

\begin{tabular}{lcc}
\hline Tipo de consumo & Mujer (\%) & Hombre (\%) \\
\hline Embriaguez & 7,1 & 14,7 \\
3 a más días & 48,8 & 45,1 \\
1 a 2 días & 35,1 & 31,8 \\
Ningún día & 9,0 & 8,4 \\
No indica & & \\
Beber excesivo & 54,0 & 60,2 \\
Al menos una vez & 35,4 & 31,3 \\
Solo una vez & 14,0 & 20,5 \\
Entre dos y tres veces & 3,3 & 3,1 \\
Entre cuatro y cinco veces & 1,3 & 5,3 \\
Más de cinco veces & 1,4 & 34,1 \\
Ninguna vez & 4,6 & 5,7 \\
No indica & & 58,0 \\
Consumo problemático de alcohol & 46,2 & 48,0 \\
Problemático & 53,8 & 52,0 \\
No problemático & & \\
\hline
\end{tabular}

Fuente: DEVIDA - Estudio Nacional sobre Prevención y Consumo de Drogas en Estudiantes de Secundaria 2017 


\section{Explicaciones a la convergencia}

Tradicionalmente, los hombres han consumido más alcohol que las mujeres, debiéndose en parte a roles específicos de género definidos culturalmente. Asimismo, el consumo de alcohol se asocia con una menor sanción social y permisividad en los hombres en comparación que en las mujeres. Sin embargo, la información epidemiológica ofrece alcances sobre un cambio significativo en el comportamiento de uso y concepciones sobre el alcohol en hombres y mujeres adolescentes del Perú.

Contrariamente a la idea de que una menor brecha de consumo de alcohol en mujeres y hombres ocurre en países desarrollados con mayor igualdad de género ${ }^{(19)}$, las prevalencias y patrones desadaptados identificados dejan clara constancia sobre la reducción de las diferencias en el uso de bebidas alcohólicas en la población adolescente a pesar de las desigualdades de género existentes en nuestro país. Es posible que ciertas condiciones culturales asociadas a un cambio en los estereotipos y roles de género hayan contribuido a este fenómeno. Del mismo modo, la mayor exposición al alcohol en las adolescentes a través de las interacciones sociales con adolescentes varones ha generado un entorno de aceptabilidad social para el consumo femenino. De esta forma, al parecer, ha disminuido la percepción negativa asociada al consumo de alcohol en las mujeres adolescentes

Otro aspecto importante a considerar es la influencia de la publicidad y la comercialización en el cambio de los estereotipos de género asociados al alcohol. A través de diversas estrategias de marketing, la industria del alcohol llega al mercado femenino en un contexto de escasa regulación y en pleno proceso de cambio en los roles de género, donde las mujeres peruanas van asumiendo un mayor empoderamiento. Aunque estas estrategias se dirigen a la población juvenil y adulta, existe evidencia de que la publicidad del alcohol y su presencia en medios de comunicación incrementa la probabilidad de que los adolescentes comiencen a consumir esta droga ${ }^{(20)}$. De acuerdo a esto, se estaría configurando un escenario propicio de acceso y normalización del consumo de alcohol desde la adolescencia para ambos géneros. Se necesita de mayor investigación (cuantitativa y cualitativa) que explore estas y otras posibles explicaciones detrás de la convergencia género en el consumo de alcohol.

\section{RETOS PARA EL \\ ESTABLECIMIENTO DE POLÍTICAS E INTERVENCIONES ASOCIADAS AL CONSUMO DE ALCOHOL EN LA POBLACIÓN ADOLESCENTE}

\section{Mejorar la cobertura y calidad de los programas preventivos en el ámbito escolar}

En la actualidad, los programas preventivos del consumo de drogas llegan a un porcentaje limitado de la población escolar de nivel secundario. En el ámbito público, tomando en consideración la cifra 2018 de implementación del Programa Presupuestal de Prevención y Tratamiento (PTCD), la cobertura asciende a $27 \%$. Es decir, aproximadamente tres de cada diez escolares de nivel secundario procedente de escuelas públicas accede a programas preventivos del consumo de drogas ${ }^{(21)}$. Para las escuelas privadas no hay estimaciones de cobertura, pero es necesaria la implantación de programas o actividades preventivas considerando que sus estudiantes presentan mayores prevalencias de consumo de alcohol respecto a los estudiantes de colegios públicos ${ }^{(9)}$.

Es importante destacar que la ampliación de coberturas es insuficiente de no considerar mecanismos institucionalizados de mejora de la calidad. Ello implica emprender acciones orientadas a obtener resultados periódicos de eficacia, efectividad y eficiencia en los programas. De esta manera, la prevención no solo debe estar disponible, sino también deben generar resultados e impactos preventivos medibles en los escolares sobre la base de programas que funcionen. Bajo este marco, la adopción de estándares de calidad basados en evidencia para el diseño e implementación de programas preventivos en el ámbito escolar es una medida oportuna ${ }^{(22)}$. Asimismo, será necesaria la inclusión de un grupo de criterios específicos de calidad que promuevan la incorporación de contenidos y estrategias sensibles al género. Llegar progresivamente a un mayor número de escuelas con programas preventivos de calidad representa una política imprescindible para afianzar los esfuerzos en la reducción del consumo de alcohol y otras drogas en los escolares.

\section{Adaptación de contenidos y estrategias preventi- vas sensibles al género}

La adolescencia es un momento crítico para la prevención. Aunque a nivel de estudios específicos diversos programas han mostrado efectos positivos, los metaanálisis reportan un modesto impacto de los programas preventivos del consumo de drogas en el ámbito escolar ${ }^{(23)}$. Esto no debe hacernos suponer que la prevención es inefectiva o una mala inversión pública. Al contrario, debe hacernos reflexionar sobre la complejidad (multifactorialidad) de la problemática del consumo de drogas y la pertinencia de contar con mejores programas preventivos. Ello implica la adopción de procedimientos basados en la evidencia que influyan desde la etapa de diseño o adaptación cultural hasta la diseminación a gran escala sustentada en estudios de eficacia y efectividad.

La información epidemiológica respalda la necesidad de adoptar un enfoque específico de género en la intervención preventiva. Los programas preventivos son más efectivos si su contenido tiene en cuenta aspectos asociados al género. Para el caso de los hombres adolescentes, las intervenciones son más efectivas si se incluyen sesiones o actividades orientadas a la mejora de habilidades sociales y asertividad, mientras que la facilitación de la expresión, reducción del estrés 
y técnicas de autocontrol emocional se han mostrado más útiles en las mujeres adolescentes ${ }^{(24)}$.

Otro tema a considerar es que los programas de habilidades para la vida, que se enfocan en el uso de sustancias y se enseñan en aulas mixtas, parecen ser una adecuada estrategia de intervención contra el consumo de alcohol en mujeres y hombres en la adolescencia temprana. En la adolescencia media y tardía, sin embargo, las diferencias de género pueden ser más pronunciadas, requiriéndose con mayor fuerza componentes específicos al género ${ }^{(25)}$.

Además, existe evidencia sobre los efectos diferenciales de programas preventivos en el ámbito escolar según el género. Así, por ejemplo, los programas preventivos basados en la mejora de habilidades psicosociales obtienen mejores resultados en las mujeres principalmente cuando se aplican a edades tempranas. Por otra parte, hombres y mujeres adolescentes pueden diferir en los efectos preventivos de las variables psicosociales de intervención del programa. Se ha observado que las adolescentes que presentaban baja autoestima tuvieron un menor beneficio. Además, se sugiere que las mujeres se encuentran más influenciadas por factores protectores familiares, mientras que los niños son más influenciados por el entorno comunitario y escolar ${ }^{(26)}$.

\section{Detección temprana, derivación e intervención breve en adolescentes con uso problemático de alcohol}

Aproximadamente 500000 escolares de nivel secundario refieren necesitar orientación sobre el consumo de drogas y 130000 señalan requerir tratamiento. La necesidad de orientación se registra en mayor medida en el grupo femenino, y la necesidad de tratamiento se reporta en mayor porcentaje en el grupo masculino. A esto debe sumarse la identificación de alrededor 67000 estudiantes que presentan beber excesivo y aproximadamente 118000 estudiantes que reportan consumo problemático de alcohol ${ }^{(9)}$. Estos datos sustentan la necesidad de reducir la brecha de cobertura de servicios de orientación y tratamiento asociado al consumo de drogas, así también como la puesta en marcha de mecanismos articulados de detección temprana, derivación e intervención breve. La implementación de estas intervenciones tendrá que considerar las características de los adolescentes, prescindiéndose de modelos tradicionales de prestación de servicios.

En nuestro país ya existe una respuesta pública que demanda ser fortalecida. Los servicios municipales de orientación sobre drogas implementados a través del PTCD, «Habla Franco» de DEVIDA, «Lugar de Escucha» de CEDRO, y los Centros de Salud Mental Comunitarios (CSMC) del Ministerio de Salud, tienen un rol fundamental en estos esfuerzos. Cabe destacar que las instituciones educativas también poseen un potencial importante de participación en las acciones de detección temprana y derivación. Para ello se deberá impartir el entrenamiento correspondiente a los docentes tutores y psicólogos. Este enfoque ha mostrado ser útil para casos que de otra forma podrían pasar desapercibidos, permitiendo la identificación de adolescentes en etapas iniciales de uso problemático de alcohol y contribuyendo al cierre de brechas entre la necesidad de tratamiento y el acceso efectivo al tratamiento ${ }^{(27,28)}$.

\section{Elaboración de guías y protocolos específicos de tratamiento para adolescentes}

Aunque no hay disponibilidad de información sobre la cantidad de adolescentes que presentan trastornos por consumo de alcohol, los datos disponibles en el Perú sobre uso problemático e intensivo de alcohol justifican la elaboración de guías y protocolos de tratamiento específicos y sensibles al género para adolescentes. Los estudios han demostrado que el tratamiento de estos problemas es eficaz para mejorar los resultados de salud en los adolescentes. La investigación metaanalítica encuentra que la terapia familiar es la intervención con mayor evidencia de efectividad en comparación a otros tipos de tratamiento ambulatorio. Sin embargo, la terapia de mejora motivacional, terapia cognitivo-conductual y la combinación de ambas también ofrecen efectos de reducción del consumo de drogas ${ }^{(29)}$.

El desarrollo de guías y protocolos deberá considerar también las barreras de tratamiento propias de la población adolescente. Por ejemplo, la investigación sobre la búsqueda de ayuda terapéutica entre los adolescentes con problemas de salud mental muestra la existencia de barreras que deben ser gestionadas por los operadores de servicios. Los adolescentes suelen mantener sus problemas para sí mismos o recurren a sus compañeros, perciben estigmatización, falta de confidencialidad, confianza limitada en los profesionales de la salud y falta de reconocimiento del problema ${ }^{(30)}$. La gestión para reducir estas barreras percibidas es fundamental para el logro de adherencia.

Vinculado a lo anterior, las guías y protocolos tendrían que ofrecer estrategias que disminuyan la posibilidad de abandono terapéutico en los adolescentes. La investigación ha identificado diversos factores que influyen en el adolescente para participar (o no) en el tratamiento: relación con los padres, percepción sobre el proceso terapéutico, calidad de la relación con el terapeuta, y expectativas sobre los resultados terapéuticos ${ }^{(31,32)}$. Lo expuesto tiene implicancias para el establecimiento de espacios sostenidos de capacitación y entrenamiento en los operadores de servicios terapéuticos.

\section{Estrategias preventivas ambientales que articulen respuestas multisectoriales y multinivel}

Una respuesta promisoria a la complejidad de los distintos factores de riesgo implicados en el consumo de alcohol es la implementación de estrategias de prevención ambiental que se orientan a modificar entornos sociales, culturales, físicos y económicos. Dado su carácter normalizado, el consumo de 
alcohol tiene un importante arraigo en estos aspectos. Por ello, una política e intervención integral sobre el consumo de alcohol en los adolescentes tendría que priorizar acciones en las comunidades, publicidad, normatividad y regulaciones.

Modificar entornos sociales y culturales que favorecen la involucración de los adolescentes en el consumo de alcohol requiere de políticas que articulen el trabajo de instituciones públicas (Ministerio de Salud, Ministerio de Educación, DEVIDA, Gobiernos Regionales, Municipalidades) y privadas (organizaciones no gubernamentales, universidades, empresas). La evidencia científica puede ofrecer orientación para una gestión multisectorial y multinivel (nacional, regional, local) efectiva.

Existe evidencia que sugiere que la exposición a mayores densidades de puntos de venta de alcohol incrementa el riesgo de uso y el uso excesivo de alcohol en los adolescentes ${ }^{(33)}$. Asimismo, la evidencia nacional muestra que los escolares de nivel secundario acceden al alcohol principalmente a través de bodegas, licorerías y supermercados ${ }^{(9)}$. La repuesta a esta coyuntura involucra acciones que pueden verse reflejadas en políticas locales que sean más estrictas para otorgar o renovar permisos de funcionamiento para los establecimientos que venden o sirven bebidas alcohólicas, restringir la presencia de publicidad exterior o vallas publicitarias de bebidas alcohólicas, prohibir el consumo público, impartir capacitación para el personal de establecimientos que venden o sirven alcohol (bodegueros, personal que atiende en bares o discotecas), entre otros. La adopción de estas medidas de forma articulada podría ejercer efectos positivos para la reducción del consumo de alcohol en los adolescentes ${ }^{(34,35)}$.

En general, se ha observado que la asunción de políticas que desarrollan mayores acciones de regulación obtiene mejores resultados en la reducción del consumo de alcohol en adolescentes. Por ejemplo, se ha observado que la probabilidad de que los estudiantes beban en el recinto escolar se incrementa cuando los estudiantes perciben una política educativa laxa o débil sobre el alcohol ${ }^{(35)}$. En correspondencia con lo anterior, existe evidencia que señala que las acciones de políticas más estrictas hacia el alcohol (tolerancia cero) presentan mejores resultados que políticas más permisivas o tolerantes ${ }^{(36,37)}$.

A nivel de intervenciones de carácter nacional para el consumo de alcohol, es necesario analizar los efectos de dos políticas: la primera es el incremento de precios/impuestos e incremento de la edad legal de consumo. Respecto a la primera, existe un cuerpo de investigación importante que indica que el aumento de precio e impuesto a las bebidas alcohólicas reduce el consumo e impactos negativos asociados ${ }^{(38,39)}$. Si bien es cierto el Ministerio de Economía y Finanzas ha realizado incrementos periódicos al impuesto selectivo al consumo para las bebidas alcohólicas, no existe información sistemática sobre los efectos de estas medidas en el consumo de alcohol en la población adulta y adolescente. Generar información en esta materia es clave para ajustar o mejorar esta política.

En cuanto a la segunda, se dispone también evidencia sobre sus resultados positivos en la disminución del uso de alcohol en adolescentes ${ }^{(40,41)}$. El Perú se encuentra en el mayoritario grupo de países que cuenta con una regulación que permite el acceso de alcohol a los mayores de 18 años. No obstante, más allá de razonamientos legales, es necesario evaluar la pertinencia de incrementar la edad mínima legal a 21 años bajo consideraciones evolutivas. Por ejemplo, la investigación establece que los cambios cerebrales estructurales continúan hasta los 20 años aproximadamente. Del mismo modo, la capacidad de toma de decisiones se desarrolla incluso luego de los 21 años de edad. El consumo de alcohol previo a esta edad pone en serio riesgo el desarrollo cerebral de los adolescentes ${ }^{(42)}$. En suma, la evidencia neurobiológica y cognitiva ofrece argumentos para considerar un incremento de la edad mínima legal.

\section{CONCLUSIÓN}

La información epidemiológica reciente sobre el consumo de alcohol en la población escolar de nivel secundario sugiere la presencia de un cambio en el patrón de uso que debe tomarse como un tema de preocupación para la salud pública, pero también como oportunidad para poner en marcha programas preventivos y terapéuticos sensibles al género y a la etapa de vida adolescente. En definitiva, la disminución de la brecha de consumo de alcohol entre mujeres y hombres adolescentes en el Perú es expresión de la naturaleza dinámica del consumo de drogas y conforma una problemática que demanda adaptabilidad y pronta respuesta desde las políticas e intervenciones.

Contribuciones de los autores: WCR ha participado en la concepción del artículo y su redacción.

Fuente de financiamiento: Autofinanciado.

Conflicto de interés: El autor declara no tener conflictos de interés.

\section{REFERENCIAS BIBLIOGRÁFICAS}

1. Squeglia LM, Jacobus J, Tapert SF. The effect of alcohol use on human adolescent brain structures and systems. Handb Clin Neurol. 2014;125:501-510. doi:10.1016/B978-0-444-62619-6.00028-8.

2. Hayatbakhsh M, Najman J, Bor W, Clavarino A, Alati R. School performance and alcohol use problems in early adulthood: a longitudinal study. Alcohol. 2011 Nov;45(7):701-9. doi: 10.1016/j.alcohol.2010.10.009.
3. Ritchwood TD, Ford H, DeCoster J, Sutton M, Lochman JE. Risky sexual behavior and substance use among adolescents: A meta-analysis. Child Youth Serv Rev. 2015;52:74-88. doi:10.1016/j.childyouth.2015.03.005

4. Mason WA, Hitch JE, Kosterman R, McCarty CA, Herrenkohl TI, Hawkins JD. Growth in adolescent delinquency and alcohol use in relation to 
young adult crime, alcohol use disorders, and risky sex: a comparison of youth from low- versus middle-income backgrounds. J Child Psychol Psychiatry. 2010;51(12):1377-1385. doi:10.1111/j.1469-7610.2010.02292.x.

5. Lester L, Baker R, Coupland C, Orton E. Alcohol misuse and injury outcomes in young people aged 10-24. J Adolesc Health. 2018;62(4):450-456. doi:10.1016/j.jadohealth.2017.10.003.

6. Bossarte R, Swahn M. The associations between early alcohol use and suicide attempts among adolescents with a history of major depression. Addict Behav. 2011;36(5):532-5. doi: 10.1016/j.addbeh.2010.12.031.

7. Hingson R, Heeren T, Winter M. Age at drinking onset and alcohol dependence: Age at onset, duration, and severity. Archives of Pediatrics \& Adolescent Medicine. 2006; 160(7), 739-746

8. World Health Organization. Global status report on alcohol and health 2018. World Health Organization, 2018.

9. Comisión Nacional para el Desarrollo y Vida sin Drogas. Estudio nacional sobre prevención y consumo de drogas en estudiantes de secundaria 2017. Lima: DEVIDA; 2019.

10. Shulte M, Ramo D, Brown S. Gender differences in factors influencing alcohol use drinking progression among adolescents. Clin Psychol Rev. 2009; 29: 535-547.

11. Erol A, Karpyak V. Sex and gender-related differences in alcohol use and its consequences: Contemporary knowledge and future research considerations. Drug Alcohol Depend. 2015 Nov 1;156:1-13. doi: 10.1016/j.drugalcdep.2015.08.023.

12. Devries K, Child J, Bacchus L, Mak J, Falder G, Graham K, Watts C, Heise L. Intimate partner violence victimization and alcohol consumption in women: a systematic review and meta-analysis. Addiction. 2014;109(3):379-91. doi: 10.1111/add.12393.

13. Slade T, Chapman C, Swift W, Keyes K, Tonks Z, Teeson M. Birth cohort trends in the global epidemiology of alcohol use and alcohol-related harms in men and women: Systematic review and metaregression. BMJ Open. 2016; 6: e011827. doi: 10.1136/bmjopen-2016-011827.

14. Comisión Interamericana para el Control del Abuso de Drogas (CICAD), Organización de los Estados Americanos (OEA). Informe sobre el consumo de drogas en las américas 2019, Washington, D.C.: CICAD; 2019.

15. Hu H, Eaton W, Anthony J, Wu LT, Cottler L. Age of first drunkenness and risks for all-cause mortality: A 27-year follow-up from the epidemiologic catchment area study. Drug Alcohol Depend. 2017;176:148-153. doi:10.1016/j.drugalcdep.2017.03.015.

16. Kuntsche E, Rossow I, Simons-Morton B, Bogt T, Kokkevi A, Godeau E. Not early drinking but early drunkenness is a risk factor for problem behaviors among adolescents from 38 European and North American countries. Alcohol Clin Exp Res. 2013;37(2):308-314. doi:10.1111/j.1530-0277.2012.01895.x.

17. Kuntsche E, Kuntsche S, Thrul J, Gmel G. Binge drinking: Health impact, prevalence, correlates and interventions. Psychol Health. 2017;32(8):976-1017. doi: 10.1080/08870446.2017.1325889.

18. Addolorato G, Vassallo GA, Antonelli G, et al. Binge drinking among adolescents is related to the development of alcohol use disorders: results from a cross-sectional study. Sci Rep. 2018;8(1):12624. doi:10.1038/s41598-018-29311-y.

19. Bloomfield K, Gmel G, Wilsnack S. Introduction to special issue gender, culture and alcohol Problems: a Multi-national study. Alcohol Alcohol Suppl. 2006;41(1):i3-i7. doi: 10.1093/alcalc/agl070.

20. Anderson P, de Bruijn A, Angus K, Gordon R, Hastings G. Impact of alcohol advertising and media exposure on adolescent alcohol use: a systematic review of longitudinal studies. Alcohol Alcohol. 2009;44(3):229-243. doi:10.1093/alcalc/agn115.

21. Comisión Nacional para el Desarrollo y Vida sin Drogas. Reporte de ejecución del programa presupuestal de prevención y consumo de drogas - PTCD 2018. Lima: DEVIDA; 2019.

22. Programa de Cooperación entre América Latina y la Unión Europea en Políticas sobre Drogas. Calidad y evidencia en reducción de la demanda de drogas. Marco de referencia para la acreditación de programas. Madrid: COPOLAD; 2014.

23. Foxcroft D, Tsertsvadze, A. Universal school-based prevention programs for alcohol misuse in young people. Cochrane Database Syst Rev. 2011;11(5):CD009113. doi: 10.1002/14651858.CD009113
24. Schinke S. Prevention science and practice: An agenda for action. J Prim Prev. 1994;15(1):45-57. doi: 10.1007/BF02196346.

25. Weichold K, Brambosch A, Silbereisen R. Do girls profit more? Gender-specific effectiveness of a life skills program against alcohol consumption in early adolescence. J Early Adolesc. 2012; 32 (2): 200-225. doi:10.1177/0272431610384489.

26. Kumpfer K, Smith P, Summerhays JF. A wakeup call to the prevention field: Are prevention programs for substance use effective for girls? Subst Use Misuse. 2008;43(8-9):978-1001. doi: 10.1080/10826080801914261.

27. Mitchell S, Gryczynski J, O'Grady K, Schwartz R. SBIRT for adolescent drug and alcohol use: current status and future directions. J Subst Abuse Treat. 2013; 44(5):463-472. doi:10.1016/j.jsat.2012.11.005.

28. Mitchell S, Gryczynski J, Gonzales A, et al. Screening, brief intervention, and referral to treatment (SBIRT) for substance use in a school-based program: Services and outcomes. Am J Addict. 2012;21 Suppl 1(0 1):S5-S13. doi:10.1111/j.1521-0391.2012.00299.x.

29. Tanner-Smith E, Wilson S, Lipsey M. The comparative effectiveness of outpatient treatment for adolescent substance abuse: A meta-analysis. J Subst Abuse Treat. 2013;44(2):145-158. doi:10.1016/j.jsat.2012.05.006.

30. Gulliver A, Griffiths K, Christensen H. Perceived barriers and facilitators to mental health help-seeking in young people: a systematic review. BMC Psychiatry. 2010;10:113. doi:10.1186/1471-244X-10-113.

31. Dakof G, Tejeda M, Liddle H. Predictors of engagement in adolescent drug abuse treatment. J Am Acad Child Adolesc Psychiatry. 2001; 40(3): 274-281.

32. de Haan A, Boon A, de Jong J, Hoeve M, Vermeiren R. A meta-analytic review on treatment dropout in child and adolescent outpatient mental health care. Clin Psychol Rev. 2013;33(5): 698-711. doi: 10.1016/j.cpr.2013.04.005

33. Shih RA, Mullins L, Ewing B, Miyashiro L, Tucker J, Pedersen E, Miles J, D’Amico E. Associations between neighborhood alcohol availability and young adolescent alcohol use. Psychol Addict Behav. 2015;29(4):950-9. doi: 10.1037/adb0000081.

34. Probst C, Monteiro M, Smith B, Caixeta R, Merey A, Rehm J. Alcohol policy relevant indicators and alcohol use among adolescents in Latin America and the Caribbean. J Stud Alcohol Drugs. 2018;79(1):49-57.

35. Paschall MJ, Grube JW, Thomas S, Cannon C, Treffers R. Relationships between local enforcement, alcohol availability, drinking norms, and adolescent alcohol use in 50 California cities. J Stud Alcohol Drugs. 2012;73(4):657-665. doi:10.15288/jsad.2012.73.657.

36. Evans-Whipp T, Plenty S, Catalano R, Herrenkohl T, Toumbourou J. The impact of school alcohol policy on student drinking. Health Educ Res. 2013;28(4):651-662. doi:10.1093/her/cyt068.

37. Epstein M, Bailey J, Furlong M, Catalano R, Toumbourou J. Does adolescent alcohol harm minimization policy exposure reduce adult alcohol problems? A cross-national comparison. J Adolesc Health. 2019; S1054-139X(19)30430-6. doi: 10.1016/j.jadohealth.2019.08.019.

38. Wagenaar A, Tobler A, Komro K. Effects of alcohol tax and price policies on morbidity and mortality: a systematic review. Am J Public Health. 2010;100(11):2270-2278. doi:10.2105/AJPH.2009.186007.

39. Sornpaisarn B, Shield K, Cohen J, Schwartz R, Rehm J. Can pricing deter adolescents and young adults from starting to drink: An analysis of the effect of alcohol taxation on drinking initiation among Thai adolescents and young adults. J Epidemiol Glob Health. 2015;5(4 Suppl 1):S45-57. doi: 10.1016/j.jegh.2015.05.004.

40. Wechsler $\mathrm{H}$, Nelson TF. Will increasing alcohol availability by lowering the minimum legal drinking age decrease drinking and related consequences among youths?. Am J Public Health. 2010;100(6):986-992. doi:10.2105/AJPH.2009.178004.

41. DeJong W, Blanchette J. Case closed: research evidence on the positive public health impact of the age 21 minimum legal drinking age in the United States. J Stud Alcohol Drugs. 2014;75 Suppl 17:108-15.

42. Silveri MM. Adolescent brain development and underage drinking in the United States: identifying risks of alcohol use in college populations. Harv Rev Psychiatry. 2012;20(4):189-200. doi:10.3109/106732 29.2012.714642. 